\title{
Dramatic Improvement of Severe Cicatricial Ectropion after Discontinuing Long-Term Erlotinib Therapy in a Patient with Lung Cancer
}

\author{
(1) Mehmet Serhat Mangan \\ Haydarpaşa Numune Training and Research Hospital, Sadık Eratik Eye Clinic, İstanbul, Turkey
}

\begin{abstract}
There is no consensus on the choice of systemic and ophthalmic treatment for patients who develop ocular toxicity with erlotinib in the few cases reported previously. Various ocular complications related to erlotinib have been reported, with one of the most serious being corneal perforation. Our patient was at risk of potential corneal perforation because of severe cicatricial ectropion and diffuse punctate corneal epitheliopathy. Therefore, erlotinib treatment was temporarily discontinued with the approval of the oncology department and the patient was closely followed. She was prescribed steroid eye ointment, single-use preservative-free artificial tears, and eye lubricant gel to protect the ocular surface. On day 4 of treatment, the patient's findings were significantly improved. After 1 week, the cicatricial ectropion had dramatically improved and the patient's complaints were completely resolved. To our knowledge, there is no case report of a patient with both ocular toxicity after long-term use that shows dramatic improvement with drug cessation, and severe cicatricial ectropion affecting the entire lower eyelid. Here, we described a patient who used erlotinib for 3 years due to non-small cell lung cancer and developed severe cicatricial ectropion which improved dramatically within one week of temporarily discontinuing erlotinib and discussed the possible reasons. Although ocular complications with erlotinib are usually encountered early in treatment, it should be kept in mind that erlotinib-related ocular complications may also arise with long-term use.
\end{abstract}

Keywords: Ectropion, cicatricial ectropion, ocular toxicity, lung cancer, erlotinib, long-term erlotinib

\section{Introduction}

Erlotinib is a tyrosine kinase inhibitor that specifically targets epidermal growth factor receptor (EGFR). ${ }^{1}$ It is frequently used in the treatment of lung cancer and may cause ocular toxicity. ${ }^{1,2,3,4}$ Ocular complications range widely from mild dry eye syndrome to corneal perforation that requires corneal transplantation. ${ }^{1,2,3,4}$ It is reported in the literature that erlotinib-related ocular complications usually occur early in treatment (within the first 6 weeks) and resolve slowly (after 6 weeks). ${ }^{1,2,3,4}$ To our knowledge, there is no case report of a patient with both ocular toxicity after long-term use that shows dramatic improvement with drug cessation, and severe cicatricial ectropion causing eversion of the entire lower eyelid. Furthermore, there is no consensus regarding the choice of systemic and ophthalmic treatment in patients who develop ocular toxicity with erlotinib in previous studies. ${ }^{1,2,3,4}$ Here, we described a patient who used erlotinib for 3 years due to non-small cell lung cancer and developed severe cicatricial ectropion which improved dramatically within one

Address for Correspondence: Mehmet Serhat Mangan, Haydarpaşa Numune Training and Research Hospital, Sadık Eratik Eye Clinic, İstanbul, Turkey E-mail: mehmetsmangan@yahoo.com ORCID-ID: orcid.org/0000-0001-7720-9003

Received: 03.04.2021 Accepted: 20.08.2021

Cite this article as: Mangan MS. Dramatic Improvement of Severe Cicatricial Ectropion after Discontinuing Long-Term Erlotinib Therapy in a Patient with Lung Cancer. Turk J Ophthalmol 2022;52:72-74

${ }^{\circledR}$ Copyright 2022 by Turkish Ophthalmological Association Turkish Journal of Ophthalmology, published by Galenos Publishing House. 
week of temporarily discontinuing erlotinib, and we discussed the possible reasons.

\section{Case Report}

Written informed consent was obtained from the patient for the publication of this case report and any accompanying images. This case report is in compliance with the ethical principles outlined in the Declaration of Helsinki.

A 70-year-old woman was referred from the oncology clinic to our ophthalmology clinic due to complaints of burning, stinging, pain, and dryness in both eyes and outward turning of both lower eyelids (Figure 1A). Her history was significant for non-small cell lung cancer, for which she had been using erlotinib (150 mg/day) for 3 years.

On ophthalmologic examination, visual acuity was 0.8 in both eyes. Diffuse punctate epitheliopathy was detected in both corneas. Diffuse hyperemia and madarosis in the tarsal conjunctiva of both lower eyelids and severe cicatricial ectropion affecting the entire lower eyelid were observed (Figure 1A, 2A). The patient also had diffuse dry, scaling skin and desquamation on her face (Figure 1A).

The patient was at risk of potential corneal perforation because of severe cicatricial ectropion and diffuse punctate corneal epitheliopathy. Therefore, erlotinib treatment was temporarily discontinued with the approval of the oncology department and the patient was closely followed in the ophthalmology clinic. She was prescribed a steroid eye ointment (hydrocortisone acetate; Cortimycine, Abdi İbrahim, Istanbul, Turkey) to be used 4 times a day on the lower eyelids to treat the ectropion. Singleuse preservative-free artificial tears (polyvinyl alcohol; Refresh,
Allergan, Westport, Ireland) every hour and an ophthalmic gel (carbomer; Lipotears, Bausch \& Lomb, Aubenas, France) 4 times a day were also prescribed to protect the cornea. On day 4 of treatment, the patient's findings had improved significantly (Figure 1B). After 1 week, her cicatricial ectropion was dramatically improved, her eyelashes were starting to grow back, and her ocular complaints were completely resolved (Figure 1C, 2B).

\section{Discussion}

Various ocular complications related to erlotinib have been reported, with corneal perforation being among the most serious. ${ }^{1-4}$ Corneal perforation can result in irreversible vision loss despite treatment by corneal transplantation. In a healthy person, EGFR may be expressed in the epithelial tissues of the cornea and conjunctiva and can be found in tears. ${ }^{1}$ Therefore, EGFR inhibition may negatively impact the ocular surface and cornea. In addition, ectropion may cause incomplete eye closure and exposure keratopathy. The simultaneous occurrence of these conditions, as in our patient, may increase the risk of corneal perforation.

In their series of five patients, Saint-Jean et al. ${ }^{1}$ reported erlotinib-related $(150 \mathrm{mg} /$ day) ectropion in only one patient after 1 month of treatment, and the ectropion persisted despite erlotinib cessation. The authors prescribed eye lubricants and ciprofloxacin ointment, but they did not specify the active ingredient of the artificial tears and whether it was preservativefree. They also did not note the severity of ectropion. ${ }^{1}$ On the other hand, Methvin and Gausas ${ }^{2}$ used only a combination antibiotic/steroid ointment as first-line treatment for a patient
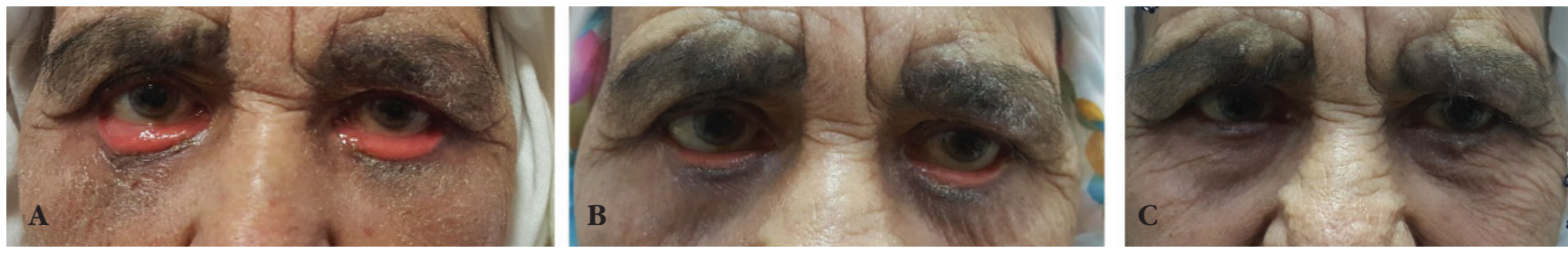

Figure 1. (A) Clinical images of the patient demonstrating severe cicatricial ectropion involving the entire lower eyelid and diffuse dry, scaling skin and desquamation on the patient's face. (B) On day 4 of treatment, the patient's findings were significantly improved. (C) After 1 week, the cicatricial ectropion was dramatically improved and the patient's ocular complaints were completely resolved
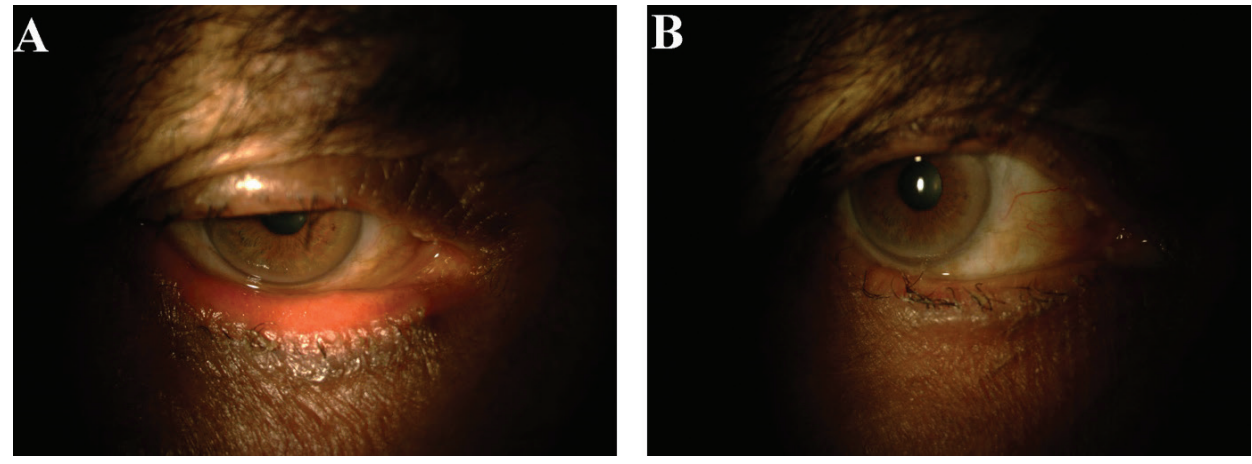

Figure 2. (A) Slit-lamp biomicroscopic photograph of the patient demonstrating lower eyelid ectropion and madarosis. (B) After 1 week, there was dramatic regression of the cicatricial ectropion and the patient's eyelashes had started to grow back 
who developed ectropion in week 6 of erlotinib $(150 \mathrm{mg} /$ day $)$ therapy. Later they reduced erlotinib from daily to every-otherday dosing but observed no improvement in the ectropion. Finally, they discontinued erlotinib and reported improvement in side effects 6 weeks later. Frankfort and Garibaldi ${ }^{3}$ observed medial ectropion 1 week after starting erlotinib $(150 \mathrm{mg} /$ day $)$ that did not improve after reducing the erlotinib dose by half (75 $\mathrm{mg} /$ day). The authors initiated bacitracin ointment and topical fluorometholone for ophthalmic treatment. When this treatment showed no effect, they added artificial tears with more frequent application of the bacitracin ointment. Later, 3 weeks after cessation of erlotinib, the findings started to improve. Salman et al. ${ }^{4}$ observed erlotinib-related mild ectropion in week 2 of treatment. They opted not to discontinue systemic erlotinib but initiated ophthalmic treatment, and reported that the ectropion was completely resolved at 6-month follow-up.

However, the authors did not specify which medications were used as ophthalmic treatment.

In contrast to these reports, our patient had severe cicatricial ectropion involving the entire lower eyelid and diffuse punctate corneal epitheliopathy. Additionally, she had been receiving erlotinib $(150 \mathrm{mg} /$ day) treatment for 3 years and the cumulative dose was much higher than in the patients reported in previous studies. ${ }^{1,2,3,4}$ Therefore, in terms of potential corneal perforation risk, we preferred to temporarily discontinue erlotinib treatment with the approval of the oncology department. Because ocular toxicity results from an inflammatory reaction, we also chose to use a potent topical steroid eye ointment at frequent intervals. We also aimed to minimize ocular surface and tear toxicity by administering preservative-free artificial tears at frequent intervals. We did not prefer an antibiotic eye ointment because there were no signs of infection and their preservative ingredients may increase ocular toxicity. We think that the dramatic improvement of severe cicatricial ectropion within one week in our patient may be due to our choice of ophthalmic treatments and good communication between the oncology and ophthalmology departments, which enabled the immediate discontinuation of erlotinib.

Our approach may reduce the need for surgical interventions to repair both the eyelid and cornea, and therefore may be preferred in cases with potential risk of corneal perforation. In addition, we think that risk assessment and mutual decisionmaking as a result of good communication between oncologists and ophthalmologists may decrease both systemic and ocular complications. Further studies with larger patient series are warranted to support this speculation. Although ocular complications with erlotinib are usually encountered early in treatment, it should be kept in mind that erlotinib-related ocular complications may also arise with long-term use.

Informed Consent: Obtained.

Peer-review: Externally peer reviewed.

Financial Disclosure: The author declared that this study received no financial support.

\section{References}

1. Saint-Jean A, Sainz de la Maza M, Morral M, Torras J, Quintana R, Molina JJ, Molina-Prat N. Ocular adverse events of systemic inhibitors of the epidermal growth factor receptor: report of 5 cases. Ophthalmology. 2012;119:17981802 .

2. Methvin AB, Gausas RE. Newly recognized ocular side effects of erlotinib. Ophthalmic Plast Reconstr Surg. 2007;23:63-65.

3. Frankfort BJ, Garibaldi DC. Periocular cutaneous toxicity and cicatricial ectropion: a potential class effect of antineoplastic agents that inhibit EGFR signaling. Ophthalmic Plast Reconstr Surg. 2007;23:496-497.

4. Salman A, Cerman E, Seckin D, Kanitez M. Erlotinib induced ectropion following papulopustular rash. J Dermatol Case Rep. 2015;9:46-48. 